\title{
Electronic structure of high- $k$ transition metal oxides and their silicate and aluminate alloys
}

\author{
G. Lucovsky, Y. Zhang, G. B. Rayner, Jr., G. Appel, and H. Ade \\ Department of Physics, North Carolina State University, Raleigh, North Carolina 27695-8202 \\ J. L. Whitten \\ Department of Chemistry, North Carolina State University, Raleigh, North Carolina 27695-8202
}

(Received 31 January 2002; accepted 13 May 2002)

\begin{abstract}
This article addresses differences between the electronic structure of: (i) alternative high- $k$ transition metal (TM) rare earth dielectrics and (ii) $\mathrm{SiO}_{2}$ and $\mathrm{Si}$ oxynitride alloys by presenting a systematic x-ray absorption spectroscopy study of transitions between TM $n p$-core states and TM metal $n$ $+1-d^{\star}$ and $n+2 s^{\star}$ antibonding/conduction band states $(n=2,3$, and 4$)$ that is complemented by studies of $\mathrm{O}$ atom $K_{1}$ edge absorption spectra. Ab initio calculations based on small clusters establish the localization of the $n+1 d^{\star}$ states on the TM metals. Ab initio electronic structure calculations are also used to interpret other aspects of the optical, ultraviolet, x-ray, and electron spectroscopies, and also provide a basis for interpretation of electrical results, thereby narrowing the field of possible replacement dielectrics for advanced semiconductor devices. (C) 2002 American Vacuum Society. [DOI: 10.1116/1.1493787]
\end{abstract}

\section{INTRODUCTION}

Noncrystalline alloys of: (i) group IIIB, IVB, and VB transition metal (TM) oxides and (ii) first row (RE) oxides with $\mathrm{SiO}_{2}$ and $\mathrm{Al}_{2} \mathrm{O}_{3}$ have been proposed as alternative gate dielectrics for advanced $\mathrm{Si}$ devices. ${ }^{1,2}$ Increases in the relative dielectric constant $(k)$ compared to $\mathrm{SiO}_{2}$ permit the use of physically thicker films to obtain the same effective capacitance as devices with physically thinner $\mathrm{SiO}_{2}$ layers thereby providing the potential for many orders of magnitude reductions in direct tunneling current. However, decreases in tunneling current anticipated from increased physical thickness are mitigated in part by reductions in effective conduction band offset energies that define the tunneling barrier between the Si substrate and high- $k$ dielectric. The nature of the these conduction band states, extended $s^{\star}$ states as in $\mathrm{SiO}_{2}$, Si oxynitride alloys, and $\mathrm{Al}_{2} \mathrm{O}_{3}$, contrasted with localized $d^{\star}$ states as in TM and RE oxides, as well their energies relative to the $\mathrm{Si}$ conduction band, are also important factors in determining the magnitude of the direct tunneling current. ${ }^{3}$ This article interprets the results of x-ray absorption spectroscopy (XAS) studies on TM and RE elemental and binary oxides in the context of ab initio calculations. The most important results of this theoretical approach include the identification of the atomic states that contribute to: (i) the highest occupied valence band states and (ii) the lowest unoccupied conduction band states of the TM/RE oxides and their silicate and aluminate alloy dielectrics. The theory identifies final-state/excitonic effects, differentiating for example between final states in intra-atom $\mathrm{x}$-ray absorption, and interlayer internal photoemission (IPE).

The local atomic bonding in noncrystalline TM/RE oxides and their silicate and aluminate alloys, and the complementary medium range order define an amorphous morphology for these dielectrics that is qualitatively different compared to $\mathrm{SiO}_{2}$ and the $\mathrm{Si}$ oxynitrides that are currently used as gate dielectrics in advanced Si devices. ${ }^{1,4}$ A framework for tracking changes in amorphous morphology from $\mathrm{SiO}_{2}$, to $\mathrm{Al}_{2} \mathrm{O}_{3}$, TM silicate and aluminate alloys, and elemental TM oxides has been developed in Ref. 1, and provides a basis for defining the local bonding coordination and symmetry of the TM atoms for the electronic structure calculations.

This classification scheme is summarized in Table I, where the character of the amorphous morphology is correlated with: (i) average bond ionicity $\left(l_{b}\right)$ using a definition due to Pauling and (ii) average oxygen atom bonding coordination. At one end of the classification scheme are idealized continuous random networks (CRNs) exemplified by $\mathrm{SiO}_{2}$, and at the other, the random closed packing ions that characterize an idealized noncrystalline structure for TM elemental oxides. ${ }^{1,4}$ Between these two limiting morphologies are modified or disrupted network structures (MCRNs) that are exemplified by bulk silicate glasses, noncrystalline silicate thin films, as well a small number of metal and TM oxides, including respectively, $\mathrm{Al}_{2} \mathrm{O}_{3}, \mathrm{TiO}_{2}$, and $\mathrm{Ta}_{2} \mathrm{O}_{5}$. ${ }^{1}$ Finally, it is significant to note that as the coordination of oxygen increases from two to four, the geometric arrangement of electrons in bonding and nonbonding orbitals remains essentially tetrahedral.

\section{SPECTROSCOPIC STUDIES}

This section presents XAS for TM/RE oxides and their pseudobinary alloys and compounds. These are interpreted in terms of the ab initio calculations presented later on in the article. The $\mathrm{Zr}$ silicate alloys, including $\mathrm{ZrO}_{2}$, and Ta and $\mathrm{Hf}$ aluminate alloys, including respectively, $\mathrm{Ta}_{2} \mathrm{O}_{5}$ and $\mathrm{HfO}_{2}$, were prepared by remote plasma enhanced chemical vapor deposition; the deposition process, and chemical and structural characterizations have been addressed in other publications. ${ }^{5-7}$ The $\mathrm{GdScO}_{3}, \mathrm{DyScO}_{3}$, and $\mathrm{LaAlO}_{3}$ 
TABLE I. Amorphous bonding morphologies for noncrystalline elemental and binary oxide alloys.

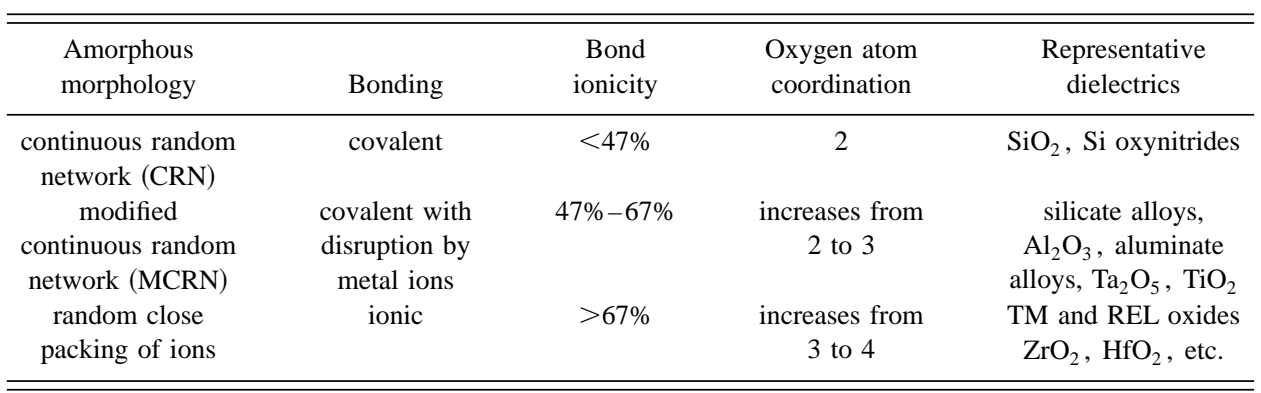

samples are single crystalline films; the preparation and characterization of these films is addressed in more detail in Ref. 8.

\section{A. Zr silicate alloys}

Figures 1 and 2 summarize the results of XAS measurements performed on $\mathrm{Zr}$ silicate alloys, $\left(\left(\mathrm{ZrO}_{2}\right)_{x}\left(\mathrm{SiO}_{2}\right)_{1-x}\right)$. Figure 1 displays XAS spectra for series of alloys with $x$ $\sim 0.2,0.5$, and 1.0, as obtained at the Brookhaven National Synchrotron Light Source; ${ }^{9}$ details of alloy preparation and compositional and structural characterization are described in Refs. 4 and 5. Spectral features in Fig. 1 have initially been assigned to dipole-allowed transitions between $M_{2,3} 3 p$ spin-orbit split $3 p_{3 / 2}$ and $3 p_{1 / 2}$ core states of the $\mathrm{Zr}$ atoms, and empty conduction band states derived from the $N_{4,5} 4 d^{\star}$ ( $a$ and $b$, and $a^{\prime}$ and $\left.b^{\prime}\right)$ and $\mathrm{O}_{1} 5 s^{\star}-\left(c\right.$ and $\left.c^{\prime}\right)$ atomic states of $\mathrm{Zr}$; however, the $a b$ initio calculations have raised issues relative this assignment of the $c$ and $c^{\prime}$ features. The transitions from the $3 p_{3 / 2}$ states are the lower energy features, $a, b$, and $c$, and the transitions from the $3 p_{1 / 2}$ states are the higher energy features, $a^{\prime}, b^{\prime}$, and $c^{\prime}$. As-deposited alloys prepared by plasma deposition are noncrystalline and pseudobinary with $\mathrm{Si}-\mathrm{O}$ and $\mathrm{Zr}-\mathrm{O}$ bonds, but no detectable $\mathrm{Zr}-\mathrm{Si}$ bonds. After annealing at $900-1000{ }^{\circ} \mathrm{C}$ in Ar, the $x=0.5$ alloys phase separates in $\mathrm{SiO}_{2}$ with crystalline $\mathrm{ZrO}_{2}$, while the $x$

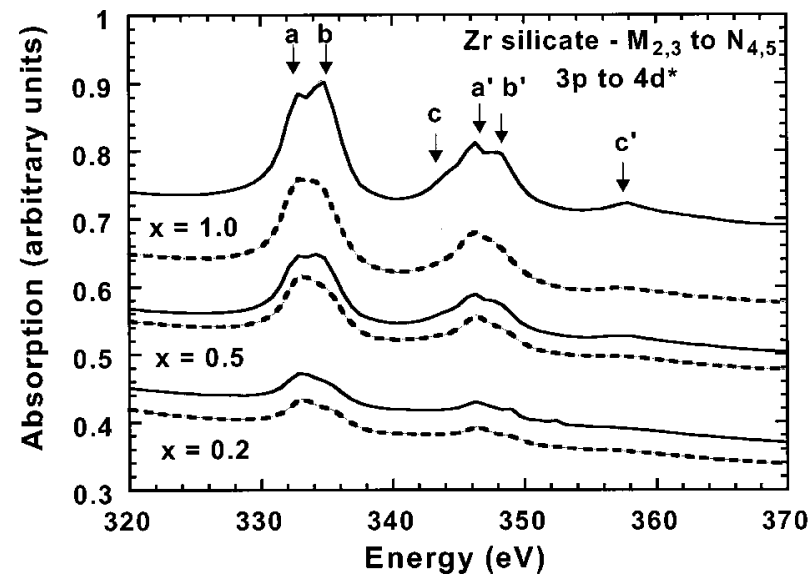

FIG. 1. XAS $M_{2,3}$ spectra for $\mathrm{Zr}$ silicate alloys indicating that features are independent of second neighbor alloy atoms, Si or Zr. $a, b$, and $c$ and $a^{\prime}, b^{\prime}$, and $c^{\prime}$ designate energy differences between the $M_{2}$ and $M_{3} p$ states, respectively, and the antibonding $\mathrm{Zr}$ states. Dotted lines are as-deposited, and dashed are after a $900{ }^{\circ} \mathrm{C}$ rapid thermal anneal.
$=1.0$ is also crystalline after annealing. ${ }^{4,5,10}$ The energies of the features highlighted in $M_{2,3}$ XAS spectra in Fig. 1 are independent, up to an experimental uncertainty of $\pm 0.2 \mathrm{eV}$, of the alloy composition and the state of crystallinity. The independence of spectral features on alloy composition, and the relatively small changes that take place upon crystallization of a $\mathrm{ZrO}_{2}$ phase after annealing are consistent with the localization of the $4 d^{\star}$ states on the $\mathrm{Zr}$ atoms. ${ }^{3}$ The spectra for the $x=0.05$ alloy (not shown in Fig. 5), and crystalline (c-) $\mathrm{ZrO}_{2}$ have been fit by Lorentzian functions with Gaussian wings, and the effective linewidths [full width at half maximum (FWHM)] of these spectral features are given in Table II.

Figure 2 displays XAS spectra for the $K_{1}$ edge of oxygen in $\mathrm{ZrO}_{2}$ and a $\mathrm{Zr}$ silicate alloy with $x \sim 0.2$; these spectra include several different groups of $\mathrm{O} 2 p^{\star}$ final states. The two lower energy bands in the $\mathrm{ZrO}_{2}$ spectrum are associated with $\mathrm{O} 2 p^{\star}$ states that are mixed with $\mathrm{Zr}$ atom $4 d^{\star}$ and $5 s^{\star}$ states. The dashed lines are the relative energies of the lowest lying antibonding states as obtained from $a b$ initio calculations. Additional structure at the high energy side of the second peak, and at energies above $540 \mathrm{eV}$, is also associated with a mixing of $\mathrm{Zr} 4 d^{\star}$ and $5 s^{\star}$ atomic states. The two features in $\mathrm{Zr}$ silicate alloy spectrum are associated in order

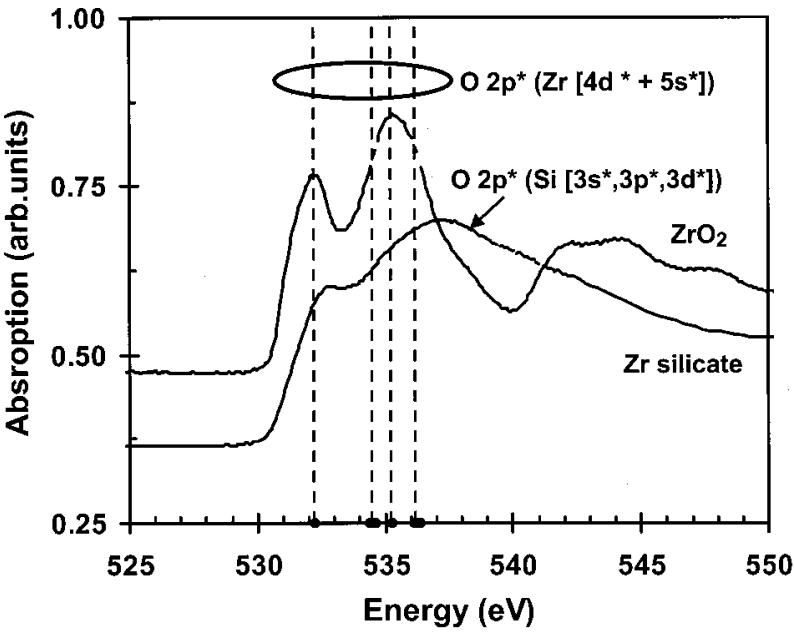

FIG. 2. O $K_{1} \mathrm{XAS}$ for $\mathrm{ZrO}_{2}$ and a $\mathrm{Zr}$ silicate alloy $(x=0.30)$. The dashed lines indicate the energies of the first two bands of $\mathrm{O} 2 p^{\star}$ states that are correlated with $\mathrm{Zr} 4 d^{\star}$ and $5 s^{\star}$ states. The low energy shoulder on the $\mathrm{Zr}$ silicate alloy spectrum is associated with $\mathrm{Zr} 4 d^{\star}$ and $5 s^{\star}$ states as well. 
TABLE II. XAS spectral features for $\mathrm{ZrO}_{2}$ and $x=0.05 \mathrm{Zr}$ silicate alloy.

\begin{tabular}{ccccc}
\hline \hline Feature & $\begin{array}{c}\mathrm{ZrO}_{2} \\
\text { energy (eV) }\end{array}$ & $\begin{array}{c}\mathrm{ZrO}_{2} \\
\text { width (ev) }\end{array}$ & $\begin{array}{c}0.05 \mathrm{ZrO}_{2} \\
\text { energy (eV) }\end{array}$ & $\begin{array}{c}0.05 \mathrm{ZrO}_{2} \\
\text { width (ev) }\end{array}$ \\
\hline $4 d$ bands: $a, a^{\prime}$ & $332.6,345.9$ & $2.65,2.65$ & $332.9,346.5$ & $2.92,2.38$ \\
$4 d$ bands: $b, b^{\prime}$ & $334.9,348.2$ & $2.72,2.72$ & $335.1,348.4$ & $2.81,2.57$ \\
$5 s$ band: $c, c^{\prime}$ & $344.4,357.8$ & $3.47,3.47$ & $344.8,358.7$ & $4.03,4.45$ \\
\hline \hline
\end{tabular}

of increasing energy with $\mathrm{O} 2 p^{\star}$ states coupled to: (i) $\mathrm{Zr} 4 d^{\star}, 5 s^{\star}$ states and (ii) $\mathrm{Si} 3 s^{\star}, s p^{\star}$ states. This assignment is based on spectra in $\mathrm{Zr}$ silicate alloys with $x$ ranging from 0.3 to 0.6 , which indicate that: (i) the difference in energy between the localized $\mathrm{Zr}$ atom $4 d^{\star}, 5 s^{\star}$ features, and the more extended $\operatorname{Si} 3 s^{\star}, p^{\star}$ states is not dependent on the alloy composition and (ii) the relative amplitudes scale with alloy composition. This interpretation is also consistent with an optical band gap of $\sim 5.5 \mathrm{eV}$ for $\mathrm{ZrO}_{2}{ }^{11}$

\section{B. $\mathrm{HfO}_{2}$ and Ta aluminate alloys}

Figures 3(a) and 3(b) present XAS results for $\mathrm{HfO}_{2}$ and a Hf aluminate alloy with $\sim 38 \% \mathrm{HfO}_{2}$, and Fig. 4 , XAS results for series of Ta aluminates with $\mathrm{Ta}_{2} \mathrm{O}_{5}$ concentrations range from 0.11 to 1.0. The spectra in Figs. 3(a) and 4 are for transitions from $N_{2,3} 4 p$ core states to $O_{4,5} 5 d^{\star}$ states, and the spectra in Fig. 4(b) are for $K_{1}$ edge of $\mathrm{O}$ for $\mathrm{HfO}_{2}$, and represent transitions to $\mathrm{O} 2 p^{\star}$ states that are coupled to Hf $5 d^{\star}$ and $6 s^{\star}$ states. Figures 3(a) and 3(b) display spectra that are qualitatively similar to those in Figs. 1 and 2 for the $\mathrm{Zr}$ silicate alloys. The most important differences between these spectra are: (i) the increased linewidth $(\sim 8 \mathrm{eV})$ and splitting $(\sim 17 \mathrm{eV})$ of the $5 d^{\star}$ state transitions, as compared to $\sim 2.5 \mathrm{eV}$ for both the width and splitting the $\mathrm{Zr} 4 d^{\star}$ state transitions, (ii) the increased spin-orbit splitting of the $N_{2,3}$ states, $\sim 56.5 \mathrm{eV}$, as compared to $\sim 13 \mathrm{eV}$ for the $M_{2,3}$ states, and (iii) the weaker low energy tails of the $N_{3}$ component are tentatively assigned the $6 s^{\star}$ analogs of the $c$ and $c^{\prime}$ features in Fig. 1. In addition, the spectra in Fig. 3(b) are quantitatively similar to those in Fig. 2. The two features in the $\mathrm{HfO}_{2}$ spectrum in Fig. 3(b) are assigned to an $\mathrm{O} 2 p^{\star}$ state mixed with the $\mathrm{Hf} 5 d^{\star}$ and $\mathrm{Hf} 6 s^{\star}$ states and are the analogs of the first two features of the $\mathrm{ZrO}_{2}$ spectrum in Fig. 2. As in the case of $\mathrm{ZrO}_{2}$, the structure at $\sim 540 \mathrm{eV}$ corresponds to transitions to $6 s^{\star}$ states. The spectrum for the $37 \% \mathrm{HfO}_{2}-\mathrm{Hf}$ aluminate alloy in Fig. 3(b) is qualitatively different from the $\mathrm{HfO}_{2}$ spectrum, but is similar to the $\mathrm{Zr}$ silicate alloy spectrum in Fig. 2. The shoulder at the edge is assigned to an $\mathrm{O} 2 p^{\star}$ state mixed with the lower $\mathrm{Hf} 5 d^{\star}$ state, and the broader feature to a mixing of the $\mathrm{O} 2 p^{\star}$ states with the second $\mathrm{Hf} 5 d^{\star}$ state, the $\mathrm{Hf} 6 s^{\star}$ state, and $\mathrm{Al} 3 s / p^{\star}$ antibonding states. Finally, the spectra in Fig. 4 for the Ta aluminate alloy series are qualitatively similar to the spectra for the $\mathrm{Zr}$ silicate alloys in Fig. 1. There is a spin-orbit replication of the two Ta $5 d^{\star}$ states, and the energies of these states are independent of the alloy composition; i.e., consistent with the localized character of the $4 p$ core states and $5 d^{\star}$ excited states. There are several quantitative differences with
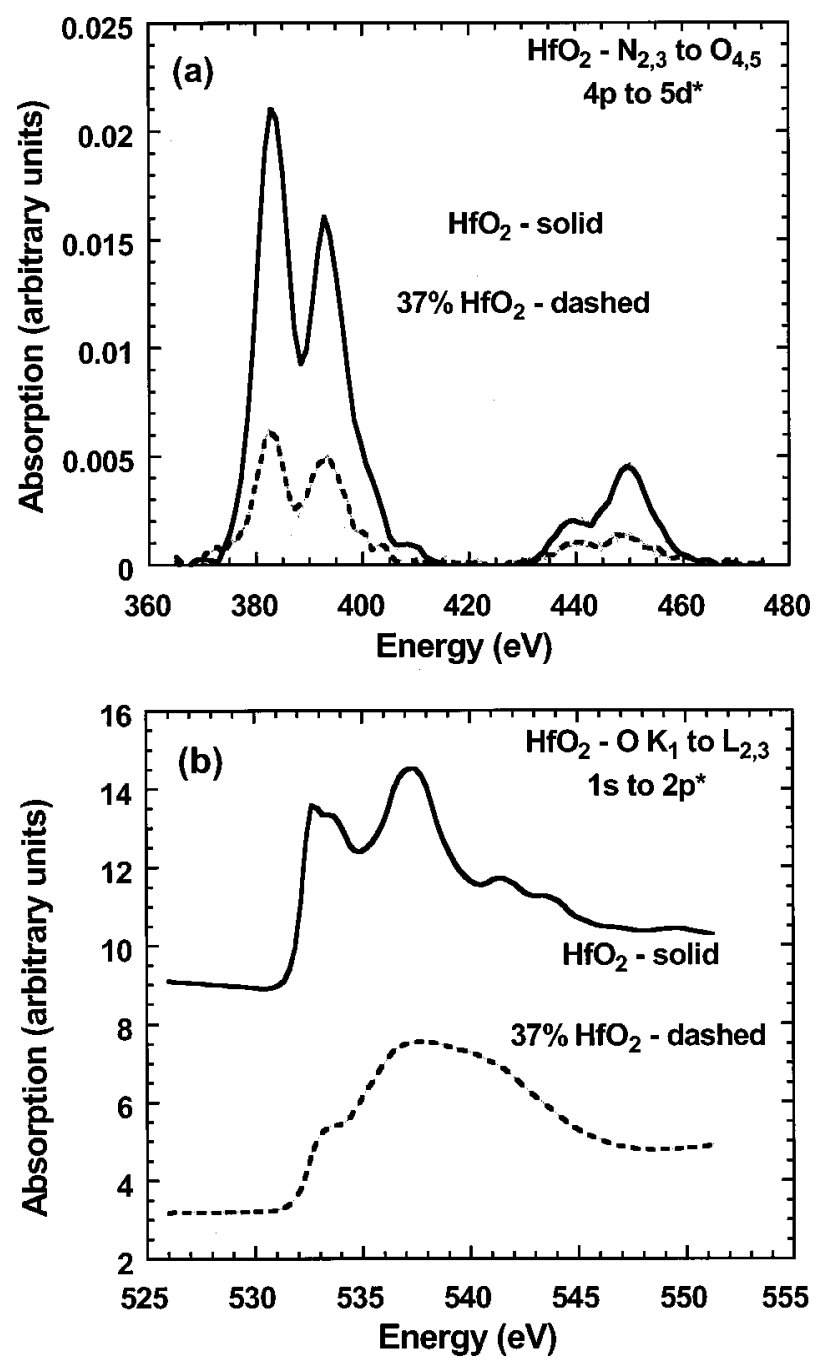

FIG. 3. (a) XAS spectra $\mathrm{Hf} N_{2,3}$ spectra for $\mathrm{HfO}_{2}$ and a $37 \%$ $\mathrm{HfO}_{2}$-aluminate alloy. The spin orbit splitting is $\sim 56.5 \mathrm{eV}$ and the FWHM $\sim 8-10 \mathrm{eV}$. (b) $\mathrm{O} L_{1} \mathrm{XAS}$ for $\mathrm{HfO}_{2}$ and a $37 \% \mathrm{HfO}_{2}$ aluminate alloy. The differences between these spectra are not due to crystallinity, but rather to changes in the conduction band spectra associated with the Al states $3 s^{\star}$ states of the alloy.

respect to the spectra in Fig. 3(a): (i) the spin-orbit splitting of the $4 p$ core states is $\sim 62 \mathrm{eV}$, similar to the spectra in Fig. 3(a) and consistent with the fact that $\mathrm{Hf}$ and Ta are next to each other in the periodic table and have approximately the same values of Z; (ii) the splitting between the two antibonding $5 d^{\star}$ states is also larger, $>15 \mathrm{eV}$, as compared to $\sim 2.5$ $\mathrm{eV}$ in Fig. 1, but comparable to the splitting of the $d^{\star}$ states of $\mathrm{Hf}$ in Fig. 3(a); and finally (iii) the widths of the $5 d^{\star}$ absorption bands are larger, $\sim 8-10 \mathrm{eV}$, as compared to $\sim 2.5$ $\mathrm{eV}$ for the spectra in Fig. 1, but comparable to the $5 d^{\star}$ state widths in Fig. 3(a).

\section{C. $\mathrm{GdScO}_{3}$ and $\mathrm{DyScO}_{3}$}

Figures 5(a) and 5(b) present XAS spectra for $\operatorname{Sc} L_{2,3} 2 p$ core states to $M_{4,5} 3 d$ state transitions in crystalline (a) $\mathrm{GdScO}_{3}$ and (b) $\mathrm{DyScO}_{3}$. The spin-orbit splitting of the Sc $2 p$ core states is reduced to $\sim 4.2 \mathrm{eV}$, and the spectral 


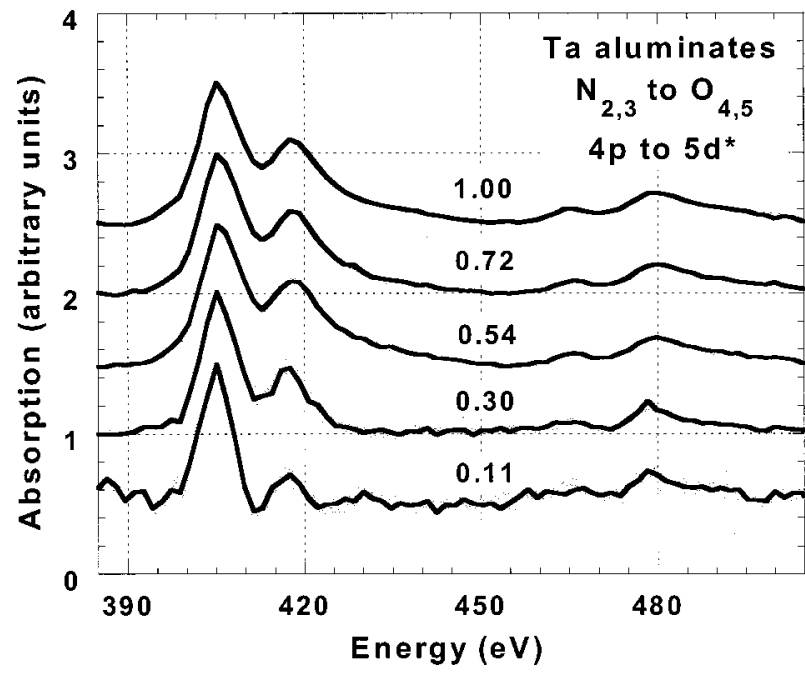

FIG. 4. XAS spectra Ta $N_{2,3}$ spectra for a series of $\mathrm{Ta}_{2} \mathrm{O}_{5}$-aluminate alloys with $x$ ranging from $0.11,0.30,0.54,0.72$, and 1.0. The lowest $\mathrm{Ta}_{2} \mathrm{O}_{5}$ content alloy is at the bottom. The spin-orbit splitting is $\sim 62 \mathrm{eV}$ and the FWHM $\sim 7-9$ eV.

linewidths are $\sim 0.4-0.5 \mathrm{eV}$. Figure 11 from Ref. 8 displays the optical absorption spectrum for $\mathrm{GdScO}_{3}$. The three features in this spectrum correspond the first $L_{2,3}$ transition in Fig. 5(a). The triplet structure, labeled $x, y, z$ in the figure, results from an orthorhombic crystal structure, and the additional triplet fine structure within each one of the crystal structure triplets is due to the off site position of the Sc atom in the perovskite structure. ${ }^{8}$ This is evidenced by the fine structure being essentially the same in the triplet features labeled $x$ and $y$, but quantitatively different in the triplet features labeled $z$. The widths of the fine-structure features are $\sim 0.080 \mathrm{eV}$.

\section{D. $\mathrm{LaAlO}_{3}$}

As noted in Ref. 8, there was no detectable XAS spectrum for transitions between La $4 p N_{2,3}$ and $5 d^{\star} O_{4,5}$ states. Other measurements presented in Ref. 8 were consistent with there being a strong overlap between the La $5 d^{\star}$ states and the next set of conduction band states, derived either from $\mathrm{Al} 3 s^{\star}$, or La $6 s^{\star}$ extended states. This overlap is consistent with the energy of the atomic La $d$ states, calculated for the $5 d^{1}, 6 s^{2}$ configuration, being closer in energy to the $6 s$ state energy than in $\mathrm{Zr}$, Hf, and Ta. ${ }^{11}$

\section{E. XAS linewidths}

The XAS data in Figs. 1, 3(a), 4, and 5(a) and 5(b) indicate systematic increases in the spectral linewidths of the $n p$ to $n+1 d$ state transitions, for $n=2,3$, and 4 , or equivalently for transitions to $3 d^{\star}, 4 d^{\star}$, and $5 d^{\star}$ states. Based on the localized nature of the $d$ state transitions, and the lack of any alloy dependence in the $\mathrm{Zr}$ silicate, and $\mathrm{Ta}$ and $\mathrm{Hf}$ aluminate alloys, the spectral linewidths of these transitions cannot be assigned to solid state bonding effects, but rather are consistent with lifetime broadening, which will be addressed below.
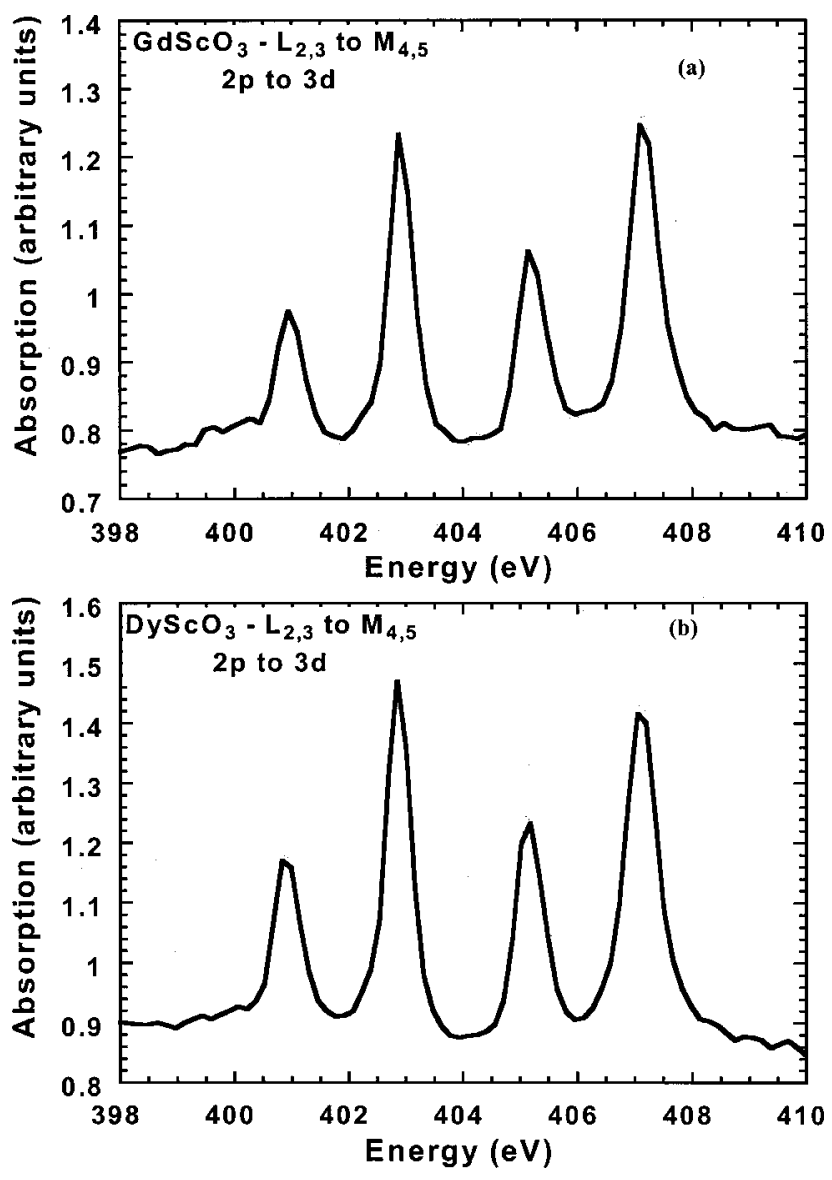

FIG. 5. XAS Sc $L_{2,3}$ spectra for: (a) $\mathrm{GdScO}_{3}$ and (b) $\mathrm{DyScO}_{3}$. The spectral features are indistinguishable. The lower two energy features are for the $2 p_{3 / 2}$ transitions, and the higher two are for the $2 p_{1 / 2}$ transitions. The spin orbit splitting is $\sim 4.5 \mathrm{eV}$ and the FWHM $\sim 0.48 \mathrm{eV}$.

\section{DISCUSSION}

This section of the article has been divided into three subsections which address: (i) a summary of the results of $a b$ initio calculations based on small clusters which provide a basis for the interpretation of the spectroscopic data of this article, as well as other studies which have addressed: (a) valence band spectra ${ }^{12,13}$ and (b) photoconductivity (PC) and IPE, ${ }^{14,15}$ (ii) interpretation of the XAS studies of this article, and (iii) interpretation of the valence band, PC, and IPE studies of Refs. 12-15. Issues relative to the correlation between spectroscopic data and electrical measurements relevant to applications of high- $k \mathrm{TM} / \mathrm{RE}$ gate dielectric materials in advanced $\mathrm{Si}$ devices will be addressed in the final section of the article.

\section{A. Ab initio calculations}

Based on previous studies which have addressed the electronic structure of TM ion complexes, ${ }^{16,17}$ the $a b$ initio results presented in this article have been based on relatively small clusters that include the TM atom at the center, and terminated O-atom neighbors. Proceeding in this way, calculations have addressed: (i) the valence band electronic structure of $\mathrm{Ti}$ and $\mathrm{Zr}$ with four and eight $\mathrm{O}$-atom neighbors, and (ii) changes in the electronic structure that are associated 


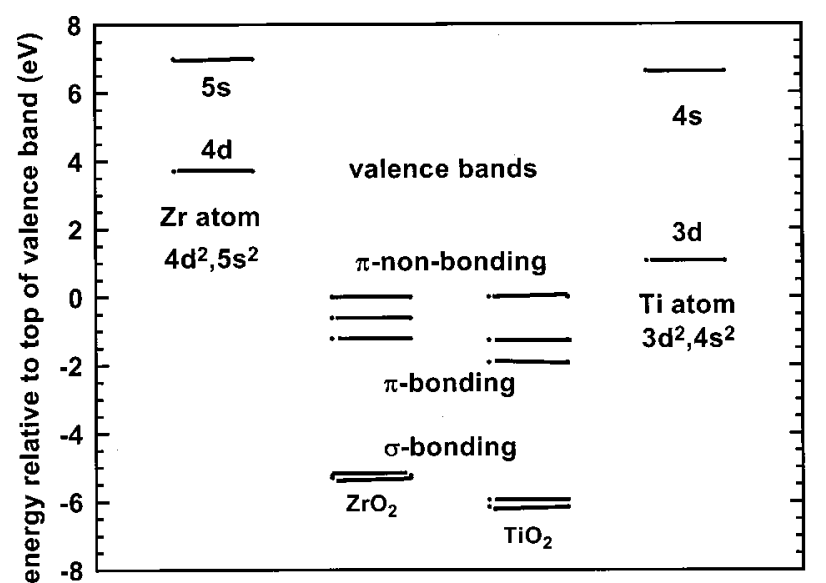

FIG. 6. Atomic state energies and valence band structures for $\mathrm{ZrO}_{2}$ and $\mathrm{TiO}_{2}$ from $a b$ initio calculations.

with: (a) excitation of the an electron from a deep $2 p$ core state to TM antibonding $d^{\star}$ and $s^{\star}$ states, (b) excitation of an electron from an $\mathrm{O}$-atom $1 s$ core state to TM antibonding $d^{\star}$ and $s^{\star}$ states, (c) excitation of an electron from an $\mathrm{O}$-atom $2 s$ valence band nonbonding state to TM antibonding $d^{\star}$ and $s^{\star}$ states, and (d) internal photoemission of electrons from a metal into the TM antibonding $d^{\star}$ and $s^{\star}$ states.

Calculations have been done at the Hartree-Fock and correlation interaction levels using exact Hamiltonians. Energy optimization has been accomplished using the variational energy calculations. Details of this approach are well documented, as for example in Refs. 18 and 19. As an example, Fig. 6 indicates the valence band states of $\mathrm{TiO}_{2}$ and $\mathrm{ZrO}_{2}$.

Figure 6 indicates ground state electronic structure for clusters containing fourfold coordinated $\mathrm{Ti}$ and $\mathrm{Zr}$ atoms, each bonded to four $\mathrm{OH}$ groups. The focus here are the atomic states of the Ti atoms in $3 d^{2} 4 s^{2}$ configurations, ${ }^{11}$ and the highest valence band states. The energies of the atomic $5 s$ states of $\mathrm{Zr}$ and $4 s$ states of $\mathrm{Ti}$ relative to the top of the valence band (arbitrarily set at $0.0 \mathrm{eV}$ ) and approximately the same, whereas the $3 d$ states of $\mathrm{Ti}$ are about $2.7 \mathrm{eV}$ closer to the valence band than those of $\mathrm{Zr}$. The highest valence band states at 0.0 are associated with nonbonding $\mathrm{O}$-atom $2 p$ states with a $\pi$ symmetry character. The next four states in each of the valence bands are associated predominantly with $\mathrm{O}$-atom $\sigma$ and $\pi$ states that are mixed respectively, with the, $\sigma$ and $\pi$ components of the TM $d$ states. That means that all five of the valence band states are highly localized on the atoms within the small cluster. The second and third valence band states have a $\pi$ bonding character and are derived from mixtures of occupied $\mathrm{O}$ atom and TM, O $2 p$ and $\mathrm{Zr} 4 d$ or Ti $3 d \pi$ states, while the more closely spaced lower conduction band states are derived from mixtures of occupied O-atom $2 p \sigma$ states and with $\mathrm{Zr} 4 d, 5 s$ or $\mathrm{Ti} 3 d, 4 s \sigma$ states.

Finally, the $a b$ initio calculations have also addressed the final state configurations corresponding to: (i) XAS transitions from TM $p$ states, and O-atom $1 s$ states, i.e., the $\operatorname{Zr} M_{2,3}$ and $\mathrm{O} 1 s K_{1}$ spectra of Figs. 1 and 2 and (ii) the optical absorption and IPE procesess, e.g., as an example, the energies of the photoemitted electron in the IPE process have

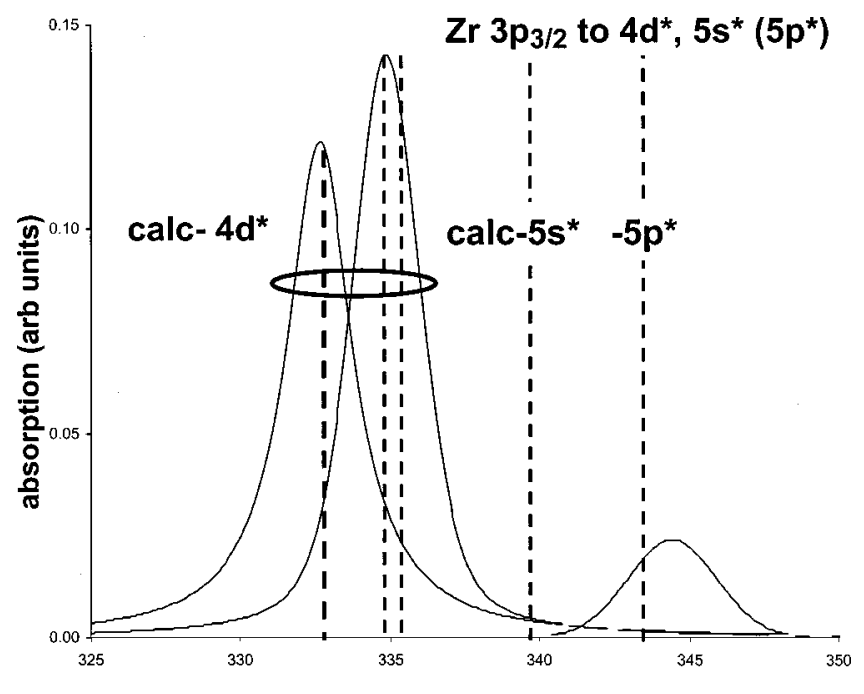

(a) energy (eV)

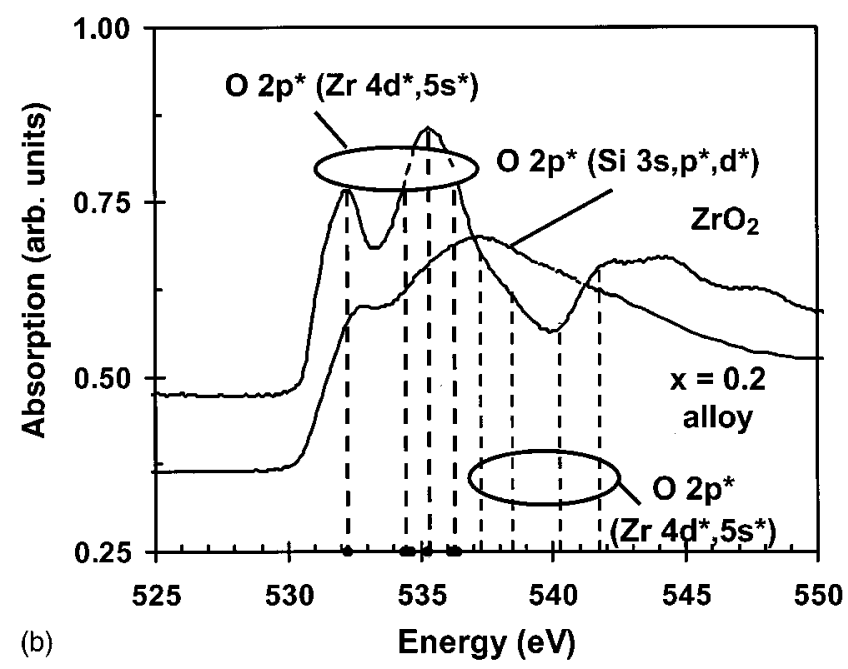

FIG. 7. (a) Deconvolution of $M_{4,5}$ spectra for $\mathrm{ZrO}_{2}$. The dashed lines indicate the results of ab initio calculations. (b) Oxygen atom $K_{1}$ spectra for $\mathrm{ZrO}_{2}$ and a $\mathrm{Zr}$ silicate alloy $(x \sim 0.20)$. The dashed lines indicate the results of $a b$ initio calculations for the $K_{1}$ spectral features.

been calculated as a function of the distance from metal electrode or silicon valence band from which the electron was emitted. This calculation is effectively an image charge calculation for an electron in a negative ion state. The potential at which the electron escapes into a transport band in the $\mathrm{Zr}$ oxide material is determined by the applied field. For fields on the order of $10^{6} \mathrm{~V} \mathrm{~cm}^{-1}$, the escape distance is about 0.5-1.0 nm, and the electron energy state more than $6 \mathrm{eV}$ above the $\mathrm{O} 2 p \pi$ nonbonding highest valence band state.

\section{B. Interpretation of the XAS results}

This emphasizes the XAS $M_{2,3}$ and $K_{1}$ spectra for $\mathrm{ZrO}_{2}$ and $\mathrm{Zr}$ silicate alloys, and also addresses the relative widths of $3 d^{\star}, 4 d^{\star}$, and $5 d^{\star}$ states. Figures $7(\mathrm{a})$ and 7(b) indicate XAS $M_{2,3}$ and $K_{1}$ spectra and include energy markers determined from the $a b$ initio calculations. Consider first the spectra in Fig. 7(a). The spectra of Fig. 1 have been deconvolved using Gaussian functions with Lorentzian wings, and the re- 
sults of this are displayed in Table II and Fig. 7(a). The deconvolved spectra in Fig. 7(a) are for the features labeled $a, b$, and $c$ in Fig. 1. The dashed lines in Fig. 7(a) are calculated relative final state energies for excitations from $\mathrm{Zr} 3 p$ to $\mathrm{Zr}$ atom $4 d^{\star}, 5 s^{\star}$, and $5 p^{\star}$ states. The $a$ and $b$ features are $4 d$ states. There is no feature at the energy corresponding to the $3 p$ to $5 s^{\star}$ transition, and this is tentatively attributed to a low transition probability associated with a change in the principal quantum number of 2 ; i.e., differences in the number of nodes in the radial part of the wave function produce significant dipole radiation matrix element cancellations. The calculated feature marked $4 d^{\star}, 5 p^{\star}$ is at the leading edge of the feature designated as $c$ in Fig. 1.

The $\mathrm{ZrO}_{2} K_{1}$ spectrum in Fig. 7(b) includes the results of the $a b$ initio calculations. The agreement between theory and experiment is excellent. In addition, these calculations indicate that the $K_{1}$ spectra display spectral features that correspond to the conduction band states that define the optical band gap, and transport states for photoconductivity. That is the calculation of final states for excitation of the $\mathrm{O}$-atom $2 p$ nonbonding states at the top of the valence band, gives essentially the same final states as for the $K_{1}$ edge calculation.

Qualitatively similar effects are anticipated for the $\mathrm{Hf} N_{2,3}$ and $K_{1}$ spectra; however spin-orbit splitting effects are anticipated due to a larger value of $Z, 72$ for $\mathrm{Hf}$ as compared to 40 for $\mathrm{Zr}$. This is consistent with the larger splitting between the $5 d^{\star}$ states in the Hf spectra compared to the $\mathrm{Zr}$ spectra, approximately $\sim 17 \mathrm{eV}$ as compared to $2.2-2.3 \mathrm{eV}$. The comparisons between the $5 d^{\star}$ state splittings and linewidths for $\mathrm{Ta}$ and Hf support this interpretation.

Finally, as noted are systematic differences between the widths and splitting of the $d^{\star}$ state features: $3 d^{\star}$ for Sc, $4 d^{\star}$ for $\mathrm{Zr}$, and $5 d^{\star}$ for $\mathrm{Hf}$ and Ta. Based on the localized nature of the $d$-state transitions, and the lack of any alloy dependence in the $\mathrm{Zr}$ silicate, and $\mathrm{Ta}$ and Hf aluminate alloys, the spectral linewidths of these transitions cannot be assigned to solid state bonding effects, but rather are consistent with lifetime broadening. Lifetime broadening is addressed in terms of the uncertainty principle in Eq. (1):

$$
\Delta W \cdot \Delta t>h / 4 \pi,
$$

where $\Delta W$ is the linewidth, $\Delta t$ is the lifetime for the transition, and $h$ is Planck's constant. The matrix element for the allowed core level transitions scales with a power $n$ of the effective nuclear charge. Figure 8 plots $\Delta W$ as a function of the nuclear charge $Z$, for $\operatorname{Sc}\{21\}, \operatorname{Zr}\{40\}$, and $\mathrm{Hf}\{72\}$, and for an effective nuclear charge $Z_{\text {eff }}$, which takes into account complete screening by inner shells, as well as partial screening by the two, three, or four $p$ states of the transition, so that $Z_{\text {eff }}=15,26$, and 40 for $\mathrm{Sc}, \mathrm{Zr}$, and $\mathrm{Hf}$, respectively. The values of $n$ obtained for the $Z$ scaling, 2.4, and $Z_{\text {eff }}$ scaling, 3.0, and about $10 \%$ larger than the values of $n$ obtained for a similar power-law fit of the spin-orbit splittings of the respective $2 p, 3 p$, and $4 p$ states as a function of $Z\left(n^{\prime}\right.$ $=2.2)$ or $Z_{\text {eff }}\left(n^{\prime}=2.7\right)$. The scaling confirms that linewidths of XAS features in Figs. 1, 3(a), 4, and 5(a) and 5(b)

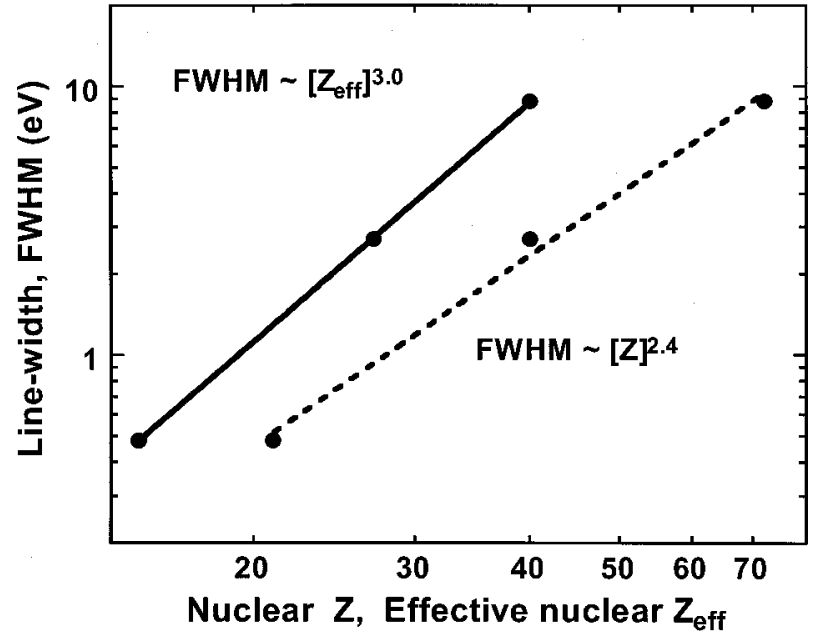

FIG. 8. Values of linewidth (FWHM) for Sc $\left(L_{2,3}\right), \mathrm{Zr}\left(M_{2,3}\right)$, and $\mathrm{Zr}\left(N_{2,3}\right)$ XAS transitions as a function of nuclear charge $(Z)$, and effective nuclear charge $\left(Z_{\text {eff }}\right)$.

are not due to solid state broadening but are correlated with strongly $Z$-dependent radiative absorption matrix elements.

\section{Interpretation of valence band, PC, and IPE results}

Figure 9 displays the valence band density of states as obtained from UPS studies of $\mathrm{TiO}_{2}{ }^{13}$ and the relative average energies of the $2 p \mathrm{O}$ atom: (i) nonbonding $\pi$ states $(0.0 \mathrm{eV})$, (ii) bonding $\pi$ states $(-1.6 \mathrm{eV}$ ), and (iii) bonding $\sigma$ states $(-6 \mathrm{eV})$ valence band features obtained from the ab initio calculations as shown in Fig. 8. The agreement between the calculated energies and spectral features is excellent. The calculations predict that the corresponding valence band states for $\mathrm{Zr}-\mathrm{O}$ bonding will be at $0.0,-0.97$, and $-5.30 \mathrm{eV}$, respectively. UPS experiments are in progress in our group to obtain the valence band density of states for $\mathrm{ZrO}_{2}$; however, measurements of Miyazaki et al. in Ref. 12 give an effective valence bandwidth for $\mathrm{ZrO}_{2}$ of approximately $5 \mathrm{eV}$ in agreement with the $a b$ initio results of Fig. 6 .

In addition to the spectroscopy studies discussed above, there have been other measurements that have addressed band gaps and band offset energies for $\mathrm{ZrO}_{2}$ and $\mathrm{HfO}_{2},{ }^{14,15}$

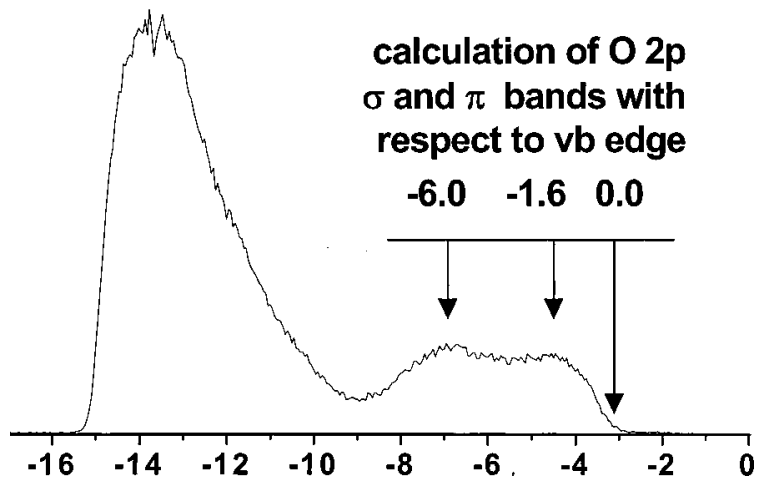

FIG. 9. Comparison between UPS valence band structure and ab initio calculation for $\mathrm{TiO}_{2}$. 
TABLE III. Final state effects in band gaps and band offset energies.

\begin{tabular}{ccccccc}
\hline \hline Measurement & $\mathrm{ZrO}_{2}(\mathrm{eV})$ & $\begin{array}{c}d \text { band } \\
(\mathrm{eV})\end{array}$ & $\begin{array}{c}\text { Conduction } \\
\text { band offset } \\
(\mathrm{eV})\end{array}$ & $\begin{array}{c}\mathrm{HfO}_{2} \\
(\mathrm{eV})\end{array}$ & $\begin{array}{c}d \text { band } \\
(\mathrm{eV})\end{array}$ & $\begin{array}{c}\text { Conduction } \\
\text { band offset } \\
(\mathrm{eV})\end{array}$ \\
\hline $\mathrm{XAS} n p$ to & 339,341 & 3.0 & -0.15 & 382,392 & - & - \\
$n+1 d(\mathrm{av})$ & & - & - & 5.8 & 5.8 & 1.55 \\
PC & - & - & 1.95 & 3.1 & 6.2 & 1.95 \\
IPE & 3.1 & 6.2 & 1.25 & 5.8 & 5.8 & 1.55 \\
XPS, UPS & 5.5 & 5.5 & &
\end{tabular}

specifically studies of PC and IPE. These are summarized in Table III, which also includes estimates of band offset energies with respect to the conduction band of $\mathrm{Si}$. Due to the spatially localized character of the $\mathrm{Zr} 4 d^{\star}$ and $\mathrm{Hf} 5 d^{\star}$ states that comprise the lowest conduction bands, the energies of these states display strong final-state or excitonic effects that depend on the location of the charge compensating hole, i.e., on the method of excitation or injection. If one takes into account differences in atomic $d$-state energies between $\mathrm{Zr}$ and $\mathrm{Hf}^{11}{ }^{11}$ then the PC band gaps of $5.5 \mathrm{eV}$ for $\mathrm{ZrO}_{2}$ in Ref. 14 , and $5.8 \mathrm{eV}$ for $\mathrm{HfO}_{2}$ in Ref. 15 are expected. This comparison assumes a rigid shift of TM $d$ states relative to the $\mathrm{O}$-atom $2 p p$ states at the top of the valence band. The rigid shift model has previously been invoked to explain scaling TM oxide optical band gaps with relative TM $d$-state energies, ${ }^{3}$ and is consistent with the seminal articles of Robertson and co-workers. ${ }^{20,21}$

The larger relative respective band offset energies for IPE for both $\mathrm{ZrO}_{2}$ and $\mathrm{HfO}_{2}$ and their nearly equal values of 6.2 $\mathrm{eV}$ relative to the $\mathrm{Si}$ valence band, are consistent with the $a b$ initio calculations in which the effective barrier for IPE is determined primarily by the applied electric field; i.e., the photoemission into continuum states with an $s^{\star}$ symmetry.

\section{CONCLUSIONS}

The section addresses four important conclusions that can made from the spectroscopic studies combined with the $a b$ initio calculations: (i) final-state or excitonic effects play a significant role in determining the energies of the TM oxide and silicate and aluminate antibonding states; (ii) the empirical tunneling models used for $\mathrm{SiO}_{2}$ and $\mathrm{Al}_{2} \mathrm{O}_{3}$ dielectrics generally cannot be applied to TM oxides, silicates, and aluminates because of the qualitatively different character of the lowest conduction band states; (iii) scaling arguments for relative band gaps of TM oxides, based on $d$-state energies are valid, but cannot be applied to silicate and aluminate alloys without taking into account changes in the coordination of the $\mathrm{O}$ atoms between the oxides and the alloys, whereas (iv) conduction band offset energies will scale with the $d$-state energies of the TM atoms.

\section{A. Final-state effects}

Final-state or excitonic effects play a significant role in determining the energies of the TM oxide and silicate and aluminate antibonding states relative the top of the valence band. This is illustrated in Table III, where the energy of the lowest antibonding $d^{\star}$ states of $\mathrm{ZrO}_{2}$ and $\mathrm{HfO}_{2}$ relative the $\mathrm{O}$-atom $2 p \pi$ nonbonding state at the top of the valence band is displayed. The effective energy difference depends on the location of the hole involved in the particular process. The XAS $M_{2,3}$ transition is intra-atomic and the offset energy is smaller than for the photoconductivity where the hole resides on an $\mathrm{O}$ atom and the transport involves $\mathrm{Zr} d^{\star}$ states. Finally, when the hole resides on a metal electrode, or in the valence band of $\mathrm{Si}$, the hole and transported electron are in different materials, on either side of an interface, and final-state effects are reduced further.

\section{B. Optical band gap scaling}

The results of this study support the previously published analysis which indicates band gap scaling with $d$-state energies in the group IV B oxides, $\mathrm{TiO}_{2}, \mathrm{ZrO}_{2}, \mathrm{HfO}_{2}$, and $\mathrm{Ta}_{2} \mathrm{O}_{5} \cdot{ }^{3,20,21}$ This scaling holds as long as there is a gap between the localized $d^{\star}$ states and $s^{\star}$ states that comprise the next highest conduction band. As noted above, this scaling is not anticipated to hold for $\mathrm{La}_{2} \mathrm{O}_{3}$, since the $5 d^{\star}$ state energies are anticipated to overlap with those of $6 s^{\star}$ states. XAS studies of $\mathrm{Y}_{2} \mathrm{O}_{3}$ are currently in progress to determine whether a similar overlap occurs. Based on atomic state energies for $\mathrm{Y}$ in a $4 d^{1} 5 s^{2}$ configuration overlap is likely to occur.

The results in Ref. 10 indicate that band gaps in TM silicate alloys decrease with increasing O-atom coordination, and that relative to the conduction and valence bands of $\mathrm{Si}$, this energy decrease will occur primarily in the valence band offset energies. The relative conduction band offset energy on the other hand is expected to be independent of alloy concentration based on the compositional dependence of XPS binding energies for $\operatorname{Si} 2 p$ and $\mathrm{Zr} 3 d_{5 / 2}$ states. $^{22}$

\section{Band offset energy scaling}

Conduction and offset energies of TM oxides have also been shown to scale with TM $d$-state energies, ${ }^{3,20,21}$ and the results of Ref. 10, indicate that this scaling is expected to persist in silicate alloys as well. What is not known and discussed below is what offset energy is appropriate for defining the barrier for electron tunneling, or whether the tunneling process is significantly modified due to the excitonic character of the $d^{\star}$ states. 


\section{Tunneling calculations}

To interpret $J-V$ tunneling data, and to predict the limitations of device scaling, it is necessary to understand the dependence of the tunneling current on the effective barrier height $E_{b}$ and the tunneling mass $m^{\star} .{ }^{23}$ The direct tunneling transmission $T$ through a rectangular barrier with a small applied voltage $V$ is given by Eq. (2):

$$
T \sim A \exp \left(-a t\left\{b\left[E_{b}-V\right] m^{\star}\right\}^{0.5}\right),
$$

where, $A, a$, and $b$ are constants, and $t$ is the physical thickness of the barrier region, i.e., the dielectric film. $E_{b}$ for the $\mathrm{SiO}_{2}$ interface is $\sim 3.1-3.2 \mathrm{eV}$, and can be determined from XPS and IPE. ${ }^{24}$ Final-state effects in $\mathrm{SiO}_{2}$ are significantly smaller than in the TM/RE dielectrics due the extended nature of the extended $\mathrm{Si} 3 s^{\star}$ states that form the lowest conduction band. The tunneling mass is approximately equal to $0.5 m_{0}$, where $m_{0}$ is the free electron mass. The $J-V$ characteristics have been calculated from self-consistent Poisson-Schroedinger equation models, and provide an excellent description of direct, as well as Fowler-Nordheim tunneling currents. This derives in part from the theory on which the models are based, including determination of the tunneling mass from extended conduction band states of $\mathrm{SiO}_{2}$. As noted above, the conduction band of $\mathrm{SiO}_{2}$ is unique among dielectrics, being derived almost exclusively from extended $\mathrm{Si} 3 s^{\star}$ antibonding states.

In addition, the basis for assigning an effective mass for the tunneling electron, and/or the manner in which the effective width of the $d$ states effects the tunneling current have not been addressed by a first principles theoretical approach from which phenomenological model equations can be derived. It is therefore not obvious how to either fit tunneling data, or how to predict tunneling currents for device structures that incorporate TM/RE high- $k$ dielectrics. The situation is even more complicated when interfacial regions with different chemical compositions are a part of a composite dielectric structure. In this regard, attempts to fit leakage currents through ultrathin TM dielectrics with tunneling equations developed for $\mathrm{SiO}_{2}$ have yielded results that are not consistent with the narrowness of the TM $d$ bands; e.g., tunneling masses in the range of $0.2 m_{0}$ have been reported for tunneling through $\mathrm{HfO}_{2}$ films in which a barrier height of $1.25 \mathrm{eV}$ was assumed. ${ }^{25}$

\section{E. Narrowing the field of high-k oxides and alloys for gate dielectric applications}

There are several criteria that can be used individually, or in combination, to remove $\mathrm{TM} / \mathrm{RE}$ dielectrics as potential candidates for device applications. These include: (i) fixed charge at $\mathrm{Si}$-dielectric interfaces, (ii) low lying $d$ states which can act as interfacial traps, transport states for leakage mechanisms other than tunneling, or low effective barriers for tunneling, and (iii) network disruption defects that act as interfacial or bulk traps.

The occurrence of increased interfacial fixed charge with respect to $\mathrm{SiO}_{2}$ is anticipated on the basis of the increased bond ionicities of the high- $k$ dielectrics, and not specifically correlated with $d$-state properties. The levels of fixed charge are $-7 \pm 1 \times 10^{12} \mathrm{~cm}^{-2}$ for $\mathrm{Al}_{2} \mathrm{O}_{3}$, and $+4 \pm 0.5$ $\times 10^{12} \mathrm{~cm}^{-2}$ for $\mathrm{ZrO}_{2} \cdot{ }^{3,26-29}$ The magnitudes of these charges are significantly less than the density of interfacial bonds at a $\mathrm{Si}(001)$ interface, $\sim 7 \times 10^{14} \mathrm{~cm}^{-2}$, suggesting that the charge arises from intrinsic bonding defects, e.g., incompletely compensated dipoles, that bridge the heterovalent interface between the covalently bonded Si substrate and the ionic dielectric. The sign of the charge, negative for $\mathrm{Al}_{2} \mathrm{O}_{3}, 3,7,26$ and positive for $\mathrm{ZrO}_{2}$ and all other oxides and silicate alloys studied to date, ${ }^{1,3}$ is consistent with the bonding of these materials. $\mathrm{Al}_{2} \mathrm{O}_{3}$ is somewhat unique in having amphoteric bonding arrangements with negatively charged, tetrahedrally bonded $\mathrm{Al}$ forming a network component that mimics $\mathrm{SiO}_{2}$, as well as sixfold coordinated $\mathrm{Al}^{3+}$ ions in a ratio of $1: 3{ }^{30}$ Finally, there is no obvious connection between the electronic structure of localized antibonding $d$ states in TM/RE dielectrics and the interfacial fixed charge.

In addition, the issue of fixed charge is multidimensional and three different aspects must be addressed: (i) fixed charge at abrupt TM/RE elemental oxide interfaces; (ii) fixed charge at abrupt TM/RE silicate alloy interfaces; and (iii) fixed charge at internal dielectric interfaces which employ ultrathin nitrided $\mathrm{SiO}_{2}$ interfacial layers with equivalent oxide thickness values $<0.3 \mathrm{~nm}$. The fixed charge at abrupt $\mathrm{TM} / \mathrm{RE}$ elemental oxide interfaces is in the mid- $10^{12} \mathrm{~cm}^{-2}$ range, and this will have an adverse effect on channel transport for a combination of channel doping and effective channel thickness in which the areal density of charged donor or acceptor atoms is less than $10^{12} \mathrm{~cm}^{-2}$.

The fixed charge at abrupt TM/RE silicate alloy interfaces is reduced in direct proportion to the concentration of the TM in the alloy. This limitation can be cast in terms of channel doping and the particular alloy composition, and the applicability to different device structures is again beyond the scope of this article. Finally, the use of nitrided $\mathrm{SiO}_{2}$ interface layers is a rather complex issue. It moves fixed charge further away from the interface as: (i) or (ii), but reduces the thickness of the high- $k$ constituent of a composite dielectric and may place additional restrictions on the tunneling leakage.

Trends in atomic $d$-state energies for $n s^{2}, n d^{n-2}$ configurations are applicable to (i) Ref. 11. In a given row, corresponding to $3 d, 4 d$, or $5 d$ electrons, the $d$-state energies become more negative as the occupancy of the $d$ states increases. This effectively limits the potential candidates to dielectrics with TM metal atoms from column III B, Sc, Y, and $\mathrm{La}, \mathrm{IV} \mathrm{B}, \mathrm{Ti}, \mathrm{Zr}$, and $\mathrm{Hf}$, and V B, V, Nb, and Ta. However, in given column, the $d$-state energies move up in energy as the effective row number increases, so that the energies of $3 d$ states are the most negative, and those of the $5 d$ states are the least negative. These two constraints eliminate $\mathrm{Sc}$ from II B, Ti from IV B, and the entire set of V B atoms, V, $\mathrm{Nb}$, and $\mathrm{Ta}$, leaving only $\mathrm{Zr}, \mathrm{Hf}, \mathrm{Y}$, and $\mathrm{La}$ as possible candidates, either as elemental oxides, or in silicate or aluminate alloys. Before addressing the network disruption defect issue, it is important to note that the $d$-state energies in the RE lanthanide series are essentially the same as $\mathrm{Y}$ and $\mathrm{La}$, so that 
the elemental oxides and silicate and aluminate alloys must also be considered as possible alternative dielectrics.

Finally, there are important differences between network disruption in silicate and aluminate alloys. ${ }^{28,29}$ Defects generated by disruption of the $\mathrm{Al}_{2} \mathrm{O}_{3}$ network component in forming TM aluminate alloys have resulted in interface traps in Hf aluminate alloys that eliminate these materials as alternative gate dielectrics as discussed in detail in Ref. 28. On the other hand, it is difficult to distinguish between traps associated with injection into Ta $d^{\star}$ states and network disruption defects in Ta aluminate alloys. ${ }^{29}$

\section{ACKNOWLEDGMENTS}

The authors acknowledge support for this research from ONR, AFSOR, DOE, SRC, and the SEMATECH/SRC Front End Processes (FEP) Center. One of the authors (G.L.) acknowledges many important interactions with colleagues in the FEP center, and in particular important insights into device requirements and measurements.

${ }^{1}$ G. Lucovsky, J. Vac. Sci. Technol. A 19, 1553 (2001).

${ }^{2}$ G. Wilk, R. W. Wallace, and J. M. Anthony, J. Appl. Phys. 89, 5243 (2001).

${ }^{3}$ G. Lucovsky, J. L. Whitten, and Yu Zhang, Microelectron. Eng. 59, 329 (2001).

${ }^{4}$ R. Zallen, The Physics of Amorphous Solids (Wiley, New York, 1983), Chap. 2.

${ }^{5}$ D. M. Wolfe, K. Flock, R. Therrien, R. Johnson, B. Rayner, L. Günther, N. Brown, B. Claflin, and G. Lucovsky, Mater. Res. Soc. Symp. Proc. 567, 343 (1999).

${ }^{6}$ G. B. Rayner, Jr., R. Therrien, and G. Lucovsky, Mater. Res. Soc. Symp. Proc. 611, c13.1 (2001).

${ }^{7}$ R. S. Johnson, G. Lucovsky, and J. Hong, J. Vac. Sci. Technol. A 19, 1353 (2001).
${ }^{8}$ S-G. Lim et al., J. Appl. Phys. (in press).

${ }^{9}$ G. Lucovsky, G. B. Rayner, Jr., D. Kang, G. Appel, R. S. Johnson, Y. Zhang, D. E. Sayers, H. Ade, and J. L. Whitten, Appl. Phys. Lett. 79, 1775 (2001).

${ }^{10}$ G. B. Rayer, Jr., D. Kang, Y. Zhang, and G. Lucovsky, J. Vac. Sci. Technol. B, these proceedings.

${ }^{11}$ W. A. Harrison, Elementary Electronic Structure (World Scientific, Singapore, 1999), Chap. 17.

${ }^{12}$ S. Miyazaki, M. Narasaki, M. Ogasawaga, and M. Hirose, Microelectron. Eng. 59, 373 (2001).

${ }^{13}$ C. C. Fulton, G. Lucovsky, and R. J. Nemanich, J. Vac. Sci. Technol. B, these proceedings.

${ }^{14}$ V. V. Afanas'ev, A. Stesmans, G. J. Andriaenssens, and M. M. Heyns, Microelectron. Eng. 59, 335 (2001).

${ }^{15}$ S. A. Campbell and V. V. Afanas'ev (unpublished).

${ }^{16}$ C. J. Ballhausen and H. B. Gray, Molecular Orbital Theory (Benjamin, New York, 1964), Chap. 8.

${ }^{17}$ P. A. Cox. Transition Metal Oxides (Oxford Science, Oxford, 1992).

${ }^{18}$ J. L. Whitten and H. Yang, Int. J. Quantum Chem., Quantum Chem. Symp. 29, 41 (1995).

${ }^{19}$ J. L. Whitten and H. Yang, Surf. Sci. Rep. 24, 55 (1996).

${ }^{20}$ J. Robertson and C. W. Chen, Appl. Phys. Lett. 74, 1168 (1999).

${ }^{21}$ J. Robertson, J. Vac. Sci. Technol. B 18, 1785 (2000).

${ }^{22}$ G. B. Rayner et al., J. Vac. Sci. Technol. B, these proceedings.

${ }^{23}$ H. Yang, H. Niimi, Y. Wu, G. Lucovsky, J. W. Keister, and J. E. Rowe, Microelectron. Eng. 48, 307 (1999).

${ }^{24}$ S. Miyazaki and M. Hirose, AIP Conf. Proc. 550, 89 (2000).

${ }^{25}$ W. Zhu, T. P. Ma, T. Tamagawa, J. Kim, R. Carruthers, M. Gibson, and T. Furukawa, Tech. Dig. - Int. Electron Devices Meet. 463 (2001).

${ }^{26}$ G. Lucovsky, J. C. Phillips, and M. F. Thorpe, AIP Conf. Proc. 550, 154 (2000).

${ }^{27}$ R. S. Johnson, G. Lucovsky, and J. Hong, J. Vac. Sci. Technol. B 19, 1606 (2001).

${ }^{28}$ R. S. Johnson, G. Lucovsky, and J. G. Hong, Microelectron. Eng. 59, 385 (2001).

${ }^{29}$ R. S. Johnson, G. Lucovsky, and J. Hong, J. Vac. Sci. Technol. A (in press.

${ }^{30}$ B. Rayner, H. Niimi, R. Johnson, R. Therrien, G. Lucovsky, and F. L. Galeener, AIP Conf. Proc. 550, 149 (2001). 